\title{
Glioblastoma Multiform: Analysis of Patients Treated with Radiotherapy at King Abdulaziz University Hospital
}

\author{
Yasir A. Bahadur ${ }^{*}$, FRCP(C) \\ Department of Radiology, Faculty of Medicine \\ King Abdulaziz University, Jeddah, Saudi Arabia \\ yasirbahadur@hotmail.com
}

\begin{abstract}
Treatment of Glioblastoma Multiform typically involves maximal surgical resection, followed by adjuvant radiation therapy. In this study, all patients with GBM treated with radiation therapy after initial surgical intervention at King Abdulaziz University Hospital were reviewed. From April, 2000 to December 2002, 13 patients were treated, $9(69 \%)$ males and $4(31 \%)$ females. Two (15\%) patients had near complete resection, one (8\%) had biopsy only and $10(77 \%)$ had partial resection. Radiation therapy was given on palliative intent in 10 (77\%) patients with dose of 24-30 Gy, and $3(23 \%)$ cases treated radically with dose of 54-60 Gy. Radiological follow-up after 6-8 weeks from completion of radiation therapy showed complete response in $8 \%$, partial response in $38 \%$, stable disease in $8 \%$, disease progression in $38 \%$ and $8 \%$ did not have their radiologic evaluation. Evaluation after one year showed overall survival rate was $36 \%$, the median survival time was 9.5 months; the median time to disease progression was 6 months. Radiation therapy provided good palliation for these patients.
\end{abstract}

Keywords: Glioblastoma multiform, Radiation therapy, Palliation.

\section{Introduction}

In spite of the substantial advances in the field of oncology due to improvements in the neuroradiology, neurosurgical techniques, and novel

\footnotetext{
*To whom all correspondence \& reprint requests: P.O. Box 80205, Jeddah 21589 Saudi Arabia. Accepted for publication: 31 December 2005. Received: 17 October 2005.
} 
methods to deliver radiation therapy, the outcome of newly diagnosed Glioblastoma Multiform (GBM) has improved little. The median survival time remains 10 to 12 months; the 2-year survival rate is under $10 \%$. The outcome in these patients continues to be related to prognostic factors more than the type of therapy ${ }^{[1]}$.

Prognosis of these patients is affected by the presence of multiple tumors and patient's related factors, like extent of disease, patient's performance status, and degree of neurological deficit. Changes in treatment protocols have so far attributed little to preventing mortality from uncontrolled local disease ${ }^{[2]}$.

The initial treatment of GBM is maximal surgical resection with minimal damage to the surrounding normal brain tissue ${ }^{[3]}$. Many trials have reported a modest improvement in local control and survival for those patients who had adjuvant radiation therapy after surgical resection when compared to those who had surgery alone ${ }^{[4]}$. A limited improvement in survival was noted in those patients who were given the addition of systemic chemotherapy to postoperative radiation therapy ${ }^{[5]}$.

The aim of this study was to review the clinical presentation and treatment results of patients with GBM referred to the radiotherapy unit of King Abdulaziz University Hospital (KAUH).

\section{Patients and Methods}

All patients with histological proven GBM treated at the Radiotherapy Unit, KAUH from April 2000 till December 2002 have been reviewed. Evaluation included patient's age, sex, performance status using Eastern Cooperative Oncology Group (ECOG) scale ${ }^{[6]}$, whether or not the patient presented with increased intracranial tension, presence or absence of cranial nerve palsy, and any neurological deficits.

Extent of disease was evaluated by computerized tomography (CT) scan or magnetic resonance imaging (MRI), criteria included: tumor size, degree of edema around the lesion and presence or absence of midline shift.

Types of surgeries included biopsy, partial resection, or complete resection. Patients with a performance status of III/IV by ECOG received postoperative palliative radiation therapy as $30 \mathrm{~Gy}$ in 10 fractions, $300 \mathrm{cGy}$ daily fractions over 2 weeks. The planning target volume was as the edema around the tumor plus 2 $\mathrm{cm}$ margin. Patients with performance status of I/II by ECOG scale received radical postoperative radiotherapy. The radiation therapy was delivered in two phases, the planning target volume for Phase One included the edema around the tumor plus $2 \mathrm{~cm}$ margin and was treated to $46 \mathrm{~Gy}$ in 23 fractions, 2 Gy per fraction per day over 4.6 weeks, then Phase Two to boost the tumor with $2 \mathrm{~cm}$ margin to a total dose of $60 \mathrm{~Gy}$. The dose was calculated by 3D using CT planning system (Cad plan) and patients were treated by Varian 2100c linear accelerator. 
Patients were followed up at 6-8 weeks after radiation therapy by CT scan / MRI to evaluate response. Complete response (CR) was defined as complete disappearance of the previously seen lesion, partial response (PR) was defined as reduction of more than $50 \%$ of the lesion, stationary disease (SD) was defined as no change or less than $50 \%$ reduction of the lesion and disease progression (DP) was defined as increase of the lesion size, or the appearance of new lesions. After the initial assessment, patients follow up was arranged every 3 months for the first 2 years, then bi-annually for the next 3 years, then per annum for 5 years. At each visit, a clinical and radiological assessment is done with the same assessment criteria mentioned before. At any time, if patients developed neurological deficits or manifestations of increased intracranial symptoms they were subjected to full clinical and radiological assessment.

Descriptive statistics were used and the overall survival (OS) was determined by Kaplan-Meier method. Overall survival was measured from the date of histological diagnosis till last follow-up or death of the patient.

\section{Results}

The total number of patients was thirteen, $9(69 \%)$ males and $4(31 \%)$ females. The median age was 51-years-old (range: 43-73 years). The performance status was III in $10(77 \%)$ patients, I in $2(15 \%)$ patients and II in one $(8 \%)$ patients. Ten $(77 \%)$ patients presented with increased intracranial pressure and $4(31 \%)$ patients had cranial nerve palsy. The average tumor size was $6 \mathrm{~cm}$ and it ranged from 2.9 to $7.5 \mathrm{~cm}$, peritumor edema was marked in 10 $(77 \%)$ patients with midline shift.

Two (15\%) patients had total gross resection of the tumor, $10(77 \%)$ had partial resection and one $(8 \%)$ patient had biopsy only. Nine $(69 \%)$ patients had palliative radiotherapy and $4(31 \%)$ had radical radiotherapy.

Radiation toxicity is summarized in Table 1.

Table 1. Radiation therapy related toxicity (Radiation Therapy Oncology Group scale).

\begin{tabular}{|c|c|c|c|c|}
\hline Toxicity & G0 & G1 & G2 & G3 \\
\hline Nausea & $22 \%$ & 0 & $54 \%$ & $24 \%$ \\
\hline Vomiting & $54 \%$ & 0 & $31 \%$ & $15 \%$ \\
\hline Headache & $46 \%$ & $15 \%$ & $31 \%$ & $8 \%$ \\
\hline Alopecia & 0 & 0 & $62 \%$ & $38 \%$ \\
\hline
\end{tabular}

Tumor response at 6-8 weeks follow-up was radiological $\mathrm{CR}$ in one $(8 \%)$ patient, PR in $5(38 \%)$, SD in $1(8 \%)$, DP in $5(38 \%)$ and one $(8 \%)$ patient had no radiological assessment.

During patients' follow-up: 4 (31\%) patients showed stable disease (SD) and $9(69 \%)$ showed disease progression (DP). At the end of follow-up, only 3 
$(23 \%)$ of 13 cases were still alive, while 10 patients $(77 \%)$ had died of their disease. Overall survival rate at one year was $36 \%$, the median survival time was 9.5 months, and the median time to disease progression was 6 months. The survival rate is shown in Fig. 1.

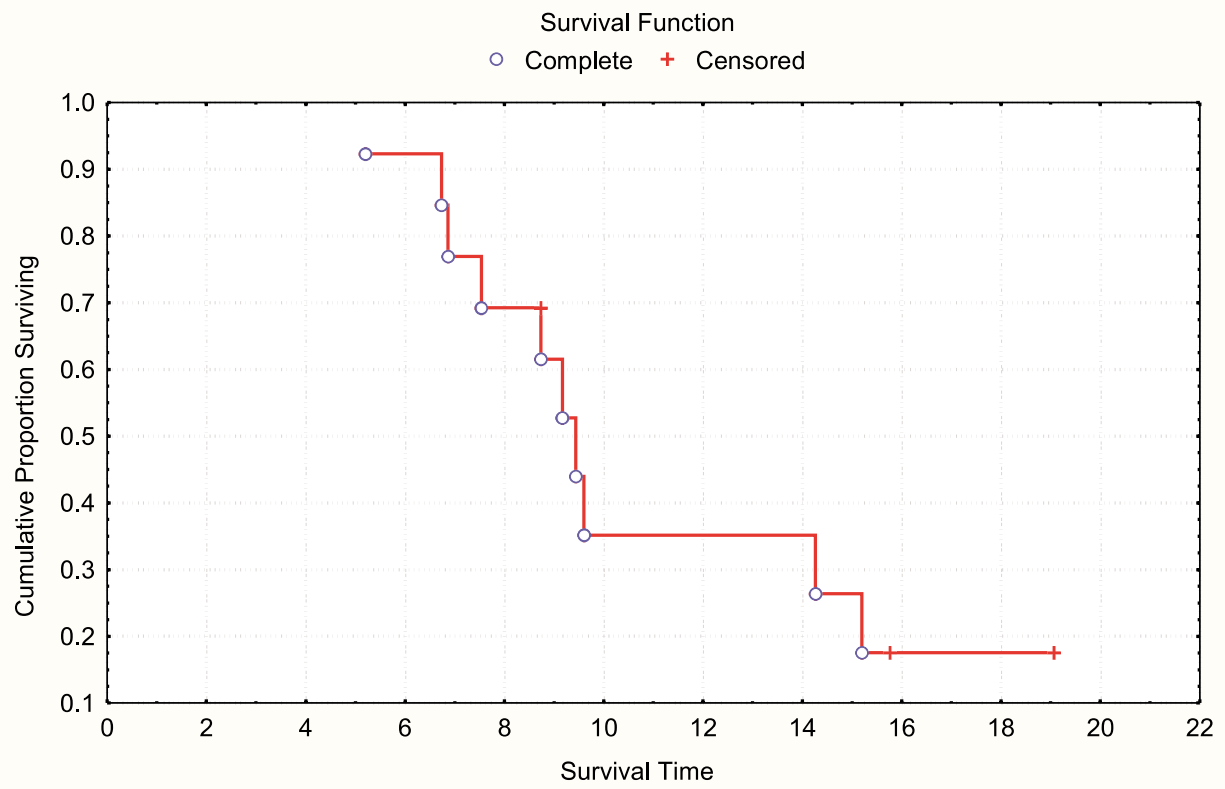

Complete: patients who died.

Censored: patients who were living on their last follow up.

Fig. 1. Overall survival curve for the patients in the study.

\section{Discussion}

GBM is considered a relatively uncommon malignancy within the overall oncology referrals to cancer centers, with an average incidence of 2.6 cases $/ 100,000$ populations per year ${ }^{[7]}$. Regional figures for its incidence showed also a low incidence rate as shown by Cancer incidence report in Saudi Arabia (year 1994-1996) ${ }^{[8]}$. The overall incidence of brain tumors was 0.2 cases $/ 100,000$ population which represented $4.4 \%$ of all cancer referrals. In Egypt; GBM cases represented only $18.2 \%$ of all gliomas cases during the period $1968-1980$ and around $32.8 \%$ during the period $1981-1991^{[9]}$. However the overall incidence of brain tumors in the same center had been found to be only $6 \%$ as compared to other types of cancer ${ }^{[10]}$. Thirteen cases of GBM were referred to the Radiotherapy Unit at KAUH during the 2 year period 2000-2002. The aim of that study was to evaluate the treatment results of patients with GBM referred to the Oncology Center at KAUH during that period. 
Surgical treatment ranged from biopsy to partial or complete surgical resection. Data from 3 prospective studies including a total of 645 patients with GBM were reviewed for the influence of extent of surgery on survival; surgical treatment consisted of biopsy only in $17 \%$ of cases, partial resection in $64 \%$, and total resection in $19 \%$ of cases; it was found that the median survival was longer for those who had either total resection (11.3 months), or partial resection (10.4 months) when compared to those who had biopsy only (6.6 months), the difference in survival among the 3 groups was not statistically significant $^{[11]}$. In Meta analysis from a series that compiled a data from 20 studies of 5,691 patients, only 4 studies found a relation between the extent of surgery and survival ${ }^{[12]}$. In the current study; the majority of cases $(77 \%)$ had partial resection.

Because of the infiltrative nature of GBM, even gross total resection is associated with a high recurrence rate, and adjuvant radiation therapy is indicated to improve local control.

Many studies demonstrate the importance of postoperative irradiation as shown by Andersen ${ }^{[13]}$ in a prospective randomized trial of patients with GBM treated with surgery only (57 cases) versus those treated with surgery and postoperative radiation therapy (51 cases); the median survival was statistically higher (23 weeks) in irradiated patients as compared to those who had surgery alone (15-weeks) $\mathrm{P}$ value $<0.005$. Walker et al. ${ }^{[4]}$, demonstrated in a prospective randomized trial that those patients treated with postoperative irradiation (93 patients) had a better median survival (36 weeks) if compared to those who had surgery alone (42 patients), whose median survival was 14-weeks, a statistically significant difference $(\mathrm{P}=0.001)$. Recently, a meta-analysis of six randomized trials detected a significant survival benefit favoring post operative radiation therapy versus no radiation therapy, risk ratio; $0.81,95 \%$ confidence interval; and $\mathrm{P}$ value $<0.0001^{[14]}$.

Before the development of conformal radiation therapy, whole cranial irradiation (WCI) was given to patients; its efficacy was evaluated by researchers such as Coffey et al. ${ }^{[15]}$ who found that those patients with GBM treated with WCI with radiation doses $>50$ Gy had a median survival of 27-weeks which was much higher than those treated with WCI at doses less than 50 Gy (11-weeks). The usual radiation dose given to patients with GBM who would be treated with WCI ranged from 50-60 Gy, and the median survival of those patients treated with doses between 50 and 60 Gy of WCI is the same as shown by Walker et al. ${ }^{[16]}$ the median survival for those patients treated with whole cranial irradiation at doses of 50, 55 and 60 Gy were 28-, 36-, and 42-weeks, respectively.

The rational of using limited field radiation therapy in GBM is that, $80-90 \%$ of cases of recurrent GBM after WCI were located within $2 \mathrm{~cm}$ of the original tumor $^{[17]}$. 
Sheline et al. ${ }^{[18]}$ reported a median survival of 10-months for GBM using limited volume radiation therapy which was comparable with that seen in patients treated with whole cranial irradiation. Wallner et al. ${ }^{[19]}$ documented that more than $90 \%$ of treatment failure were due to tumor recurrence at original site.

Radiation therapy dose when limited volume is used is $56-60 \mathrm{GY}$ in $28-30$ fractions which resulted in a one year survival of 39\% and 18-month survival of $18 \%[20]$. Dose escalation above $60 \mathrm{~Gy}$ did not result in any improvement in disease control or survival as shown by Scott et al. ${ }^{[21]}$ compared in a randomized prospective study 520 patients with GBM who received either 72 Gy / 60 fractions / 6 weeks (hyper-fractionated arm) or $60 \mathrm{~Gy} / 30$ fractions / 6 weeks (standard arm); the median survival for the two groups was 11 months and 12.2 months, respectively $(\mathrm{P}$ value $=0.44)$.

Chan et al. ${ }^{[22]}$ treated 34 patients with supratentorial GBM with 90 Gy/45 fractions after initial surgical resection using 3 dimensional treatment planning system, not including peritumoral edema in the gross tumor volume (GTV), and the planning target volume (PTV) was designed with $2.5 \mathrm{~cm}$ around the GTV, using intensity modulated radiation therapy technique and giving the radiation through 5-6 non-coplanar fields. The median survival was only 11.7 months and the 1- and 2-year survival rate was $47.1 \%$ and $12.9 \%$, respectively.

Chemotherapy has been used in GBM, in the Brain Tumor Study Group (BTSG) trial (protocol 69-01) patients post-surgery had either supportive care, carmustine, whole cranial irradiation (WCI), or WCI and carmustine. The median survival for the two groups of patients who had WCI and WCI with carmustine was identical ${ }^{[4]}$. Deutsch et al. ${ }^{[23]}$ reported BTSG protocol 77-02, 603 patients with GBM were randomized between 4 arms post surgery: arm I - carmustine plus conventional radiotherapy $(60 \mathrm{~Gy} / 30$ fractions/ 6 weeks); arm II streptozocin plus conventional radiotherapy, arm III: carmustine and hyper-fractionated radiotherapy (66 Gy /60 fractions given as 1.1 Gy twice daily (BID) over 6 weeks); and arm IV - conventional radiotherapy with misonidazole as radiosensitiser followed by carmustine. The median survival of the 4 arms was similar about 10 -months. Prados et al. ${ }^{[24]}$ in a prospective randomized trial on 231 cases of GBM tried an accelerated hyperfractionation (70.4 Gy /44 fractions, 1.6 Gy per fraction given as twice daily fractions) alone or with chemotherapy (DiFluoroMethylOrnithine - DFMO) vs. conventional radiation given as $59.4 \mathrm{~Gy} / 33$ fractions, $1.8 \mathrm{~Gy}$ per fraction alone or with DFMO, the median survival for the 4 groups of patients were 10-, 10.5-, 9.2-, and 4-months, respectively. The difference was not statistically significant $(\mathrm{P}=$ $0.48)$. Other studies confirmed the lack of benefit from chemotherapy ${ }^{[10,25]}$.

Previously mentioned studies showed that aggressive treatment modalities were not of additional benefit to these patients. Thus, a hypofractionated schedule of radiation therapy was adopted for these patients, considering the 
palliative nature of treatments and the aggressive course of the disease. Hoegler and Davey ${ }^{[26]}$ and Thomas et al. ${ }^{[27]}$ used palliative radiation therapy course of $30 \mathrm{~Gy} / 6$ daily fractions and $30 \mathrm{~Gy} / 10$ daily fractions respectively, the median survival ranged beween 4-8 months with an acceptable toxicity profile; results similar to those reported with prolonged fractionations schedules ${ }^{[18,19,28]}$ in a prospective randomized trial which compared 3 different fractionation schedules in 155 patients with GBM: either $66 \mathrm{~Gy} / 33$ fractions versus $40 \mathrm{~Gy} / 8$ fractions ( $5 \mathrm{~Gy} /$ fraction), or $28 \mathrm{~Gy} / 4$ fractions ( $7 \mathrm{~Gy}$ per fraction), the median survival was 7-, 5.6-, and 6.6-months, respectively, no difference in survival was noted among the 3 groups.

The current study is a retrospective review of GBM cases treated at KAUH, $77 \%$ were treated surgically with partial resection as compared with $65 \%$ (Prados et al..$^{[2]}$ ) and 74\% (Grossman et al. ${ }^{[1]}$ ). Sixty-nine percent of patients in this study received irradiation on palliative basis ( $30 \mathrm{~Gy} / 10$ fractions) due to poor performance status (ECOG performance scale III/IV) with a median survival of 9.5 months, and a 1 year survival rate of $36 \%$. Hulshof et al. ${ }^{[28]}$ found that the median survival for patients treated with $40 \mathrm{~Gy} / 8$ fractions or 28 Gy/ 4 fractions, were 7- and 5.6-months, respectively. McAleese et al. ${ }^{[30]}$ in a comparative study of hypofractionated radiation (using $30 \mathrm{~Gy} / 6$ fractions / 2 weeks) to GBM patients versus conventional method of $60 \mathrm{~Gy} / 30$ fractions $/ 6$ weeks found that median survival was higher among the conventional arm but was not statistically significant. Chang et al. ${ }^{29]}$ in a higher dose schedule (50 Gy/20 fractions at 2.5 Gy per fraction) found that the median survival was 7 months. In centers that treat on a radical basis like Brandes et al. ${ }^{[31]}$ patients received $59.4 \mathrm{~Gy} / 33$ (1.8 Gy per fraction) either alone (arm I) or with chemotherapy procarbazine, lomustine and vincristine (arm II) or with temozolomide (arm III), the median survival of the whole group was 7.2 months without any statistically significant difference between the 3 groups.

Radiation toxicity, in the current study was acceptable with gastrointestinal Grade II (GII) nausea in 54\% of patients, vomiting GII in 31\%, headache GII in $31 \%$. The toxicity profile of hypofractionated radiation therapy in GBM has been found to be acceptable in other studies as that shown by Chang et al. ${ }^{[29]}$, and McAleese et al. ${ }^{[30]}$.

\section{Conclusion}

Hypofractionated radiation therapy is an acceptable treatment modality in patients with poor performance GBM with an acceptable toxicity profile. It is more convenient to the patient and reduces the cost of treatment. It is recommended to study the hypofractionated irradiation in a larger number of patients with a longer follow-up. 


\section{References}

[1] Grossman SA, O'Neill A, Grunnet M, Mehta M, Pearlman JL, Wagner H, Gilbert M, Newton HB, Hellman R, Eastern Cooperative Oncology Group. Phase III study comparing three cycles of infusional carmustine and cisplatin followed by radiation therapy with radiation therapy and concurrent carmustine in patients with newly diagnosed supratentorial glioblastoma multiforme: Eastern Cooperative Oncology Group Trial 2394. J Clin Oncol 2003; 21(8): 1485-91.

[2] Andratschke N, Grosu AL, Molls M, Nieder C. Perspectives in the treatment of malignant gliomas in adults. Anticancer Res 2001; 21(5): 3541-50.

[3] Berger MS. Malignant astrocytomas: surgical aspects. Semin Oncol 1994; 21(2): 172-185.

[4] Walker MD, Alexander E Jr, Hunt WE, MacCarty CS, Mahaley MS Jr, Mealey J Jr, Norrell HA, Owens G, Ransohoff J, Wilson CB, Gehan EA, Strike TA. Evaluation of $\mathrm{BCNU}$ and/or radiotherapy in the treatment of anaplastic gliomas. A cooperative clinical trial. J Neurosurg 1978; 49(3): 333-343.

[5] Stewart LA. Chemotherapy in adult high-grade glioma: a systematic review and meta-analysis of individual patient data from 12 randomized trials. Lancet 2002; 359(9311): 1011-1018.

[6] Extermann M, Overcash J, Lyman GH, Parr J, Balducci L. Comorbidity and functional status are independent in older cancer patients , J Clin Oncol 1998; 16(4): 1582-1587.

[7] Mineo JF, Quintin-Roue I, Lucas B, Buburusan V, Besson G. Glioblastomas: clinical study and search for prognostic factors. Neurochirurgie 2002; 48(6): 500-509.

[8] [No authors listed]. Cancer Incidence Report, Saudi Arabia. 1994-1996. Depositary number in King Fahad National Library: 0110/16. Kingdom of Saudi Arabia. National Cancer Registry: 1999.

[9] Hussein K, El-Haddad S, El-Ghoneimy E. Radiotherapy of High Grade Astrocytoma. MD Thesis. Cairo U: 1991.

[10] Abdel-Salam Mahmoud, El-Ghoneimy E, Hasan A, Abdel Hady E. Neoadjuvant Chemotherapy and Radiotherapy for High Grade Astrocytoma. MD Thesis. Cairo U, Egypt: 1999.

[11] Simpson JR, Horton J, Scott C, Curran WJ, Rubin P, Fischbach J, Isaacson S, Rotman M, Asbell SO, Nelson JS, et al. Influence of location and extent of resection on survival of patients with glioblastoma multiforme: results of three consecutive Radiation Therapy Oncology Group (RTOG) clinical trials. Int J Radiat Oncol Biol Phys 1993; 26(2): 239-244.

[12] Quigley MR, Maroon JC. The relationship between survival and the extent of resection in patients with supratentorial malignant gliomas. Neurosurgery 1991; 29(3): 385-388.

[13] Andersen AP. Postoperative irradiation of glioblastomas. Results in a randomized series. Acta Radiol Oncol Radiat Phys Biol 1978; 17(6): 475-484.

[14] Laperriere N, Zuraw L, Cairncross G; Cancer Care Ontario Practice Guidelines Initiative Neuro-Oncology Disease Site Group. Radiotherapy for newly diagnosed malignant glioma in adults: a systematic review. Radiother Oncol 2002; 64(3): 259-273.

[15] Coffey RJ, Lunsford LD, Taylor FH. Survival after stereotactic biopsy of malignant gliomas. Neurosurgery 1988; 22(3): 465-473.

[16] Walker MD, Strike TA, Sheline GE. An analysis of dose-effect relationship in the radiotherapy of malignant gliomas. Int J Radiat Oncol Biol Phys 1979; 5(10): 1725-1731.

[17] Hochberg FH, Pruitt A. Assumptions in the radiotherapy of glioblastoma. Neurology 1980; 30(9): 907-911. 
[18] Sheline GE. Radiotherapy for high grade gliomas. Int J Radiat Biol Oncol Phys 1990; 18 (4): 793-803.

[19] Wallner KE, Galicich JH, Krol G, Arbit E, Malkin MG. Patterns of failure following treatment for glioblastoma multiform and anaplastic astrocytoma. Int $J$ Radiat Oncol Biol Phys 1989; 16(6): 1405-1409.

[20] Bleehen NM, Girling DJ, Gregor A, Leonard RC, Machin D, McKenzie CG, Morgan DA, Smyth JF, Spittle MF, Stephens RJ, et al. A Medical Research Council phase II trial of alternating chemotherapy and radiotherapy in small-cell lung cancer. The Medical Research Council Lung Cancer Working Party. The Medical Research Council Brain Tumor Working Party. Br J Cancer 1991; 64(4): 769-774.

[21] Scott CB , Curran WJ Jr, Yung WKA, Scarantino C, Urtasun R, Movsas B, et al. Long term results of RTOG 9006: A randomized study of hyperfractionated radiotherapy (RT) $t$ 72 Gy \& carmustine vs. standard RT \& carmustine fot malignant glioma patients with emphasis on anaplastic asttrocytoma (AA) patients [abstract]. Proc Annu Meet Am Soc Clin Oncol 1998; 401a: 1546.

[22] Chan J, Lee S, Fraass B, Normolle D, Greenberg H, Junck L, Gebarski S, Sandler H. Survival and failure patterns of high-grade gliomas after three-dimensional conformal radiotherapy. J Clin Oncol 2002; 20(6): 1635-1642.

[23] Deutsch M, Green SB, Strike TA, Burger PC, Robertson JT, Selker RG, Shapiro WR, Mealey J Jr, Ransohoff J 2nd, Paoletti P, et al. Results of a randomized trial comparing $\mathrm{BCNU}$ plus radiotherapy, streptozotocin plus radiotherapy, BCNU plus hyperfractionated radiotherapy, and $\mathrm{BCNU}$ following misonidazole plus radiotherapy in the postoperative treatment of malignant glioma. Int J Radiat Oncol Biol Phys 1989; 16(6): 1389-1396.

[24] Prados MD, Wara WM, Sneed PK, McDermott M, Chang SM, Rabbitt J, Page M, Malec M, Davis RL, Gutin PH, Lamborn K, Wilson CB, Phillips TL, Larson D. Phase III trial of accelerated hyperfractionation with or without difluromethylornithine (DFMO) versus standard fractionated radiotherapy with or without DFMO for newly diagnosed patients with glioblastoma multiform. Int J Radiat Oncol Biol Phys 2001; 49(1): 71-77.

[25] El Tawil AE, Mostafa H, Haggag F. Clinical Study and Results of Treatment of Glioblastome Multiform in NEMROCK. MSc Thesis, Cairo U, Egypt; 1987. 74-95.

[26] Hoegler DB, Davey P. A prospective study of short course radiotherapy in elderly patients with malignant gliomas. J Neurooncol 1997; 33(32): 201-204.

[27] Thomas R, James N, Guerrero D, Ashley S, Gregor A, Brada M. Hypofractionated radiotherapy as a palliative treatment in poor prognosis patients with high grade gliomas. Radiother Oncol 1994; 33(2): 113-116.

[28] Hulshof MC, Schimmel EC, Andries BD, Gonzalez-Gonzalez D. Hypofractionation in glioblastoma multiforme. Radiother Oncol 2000; 54(2): 143-148.

[29] Chang EL, Yi W, Allen PK, Levin VA, Sawaya RE, Maor MH. Hypofractionated radiotherapy for elderly or younger low-performance status glioblastoma patients: outcome and prognostic factors. Int J Radiat Oncol Biol Phys 2003; 56(2): 519-528.

[30] McAleese JJ, Stenning SP, Ashley S, Traish S, Hines F, Sardell S, Guerrero D, Brada M. Hypofractionated radiotherapy for poor prognosis malignant glioma: matched pair survival analysis with MRC controls. Radiother Oncol 2003; 67(2): 177-182.

[31] Brandes AA, Vastola F, Basso U, Berti F, Pinna G, Rotilio A, Gardiman M, Scienza R, Monfardini S, Ermani M. A prospective study of glioblastoma in the elderly. Cancer 2003; 97(3): 657-662. 


\title{
الورم الدبقى متعدد الأشكال : دراسة للمرضى المعالجين بالأشعة بجامعة الملك عبدالعزيز
}

\author{
ياسر عبد العزيز بهادر \\ قسم الأشعة ، كلية الطب ، جامعة الملك عبدالعزيز \\ جـــة - المملكة العربية السعودية
}

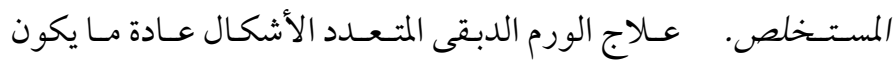

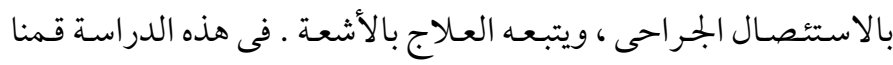

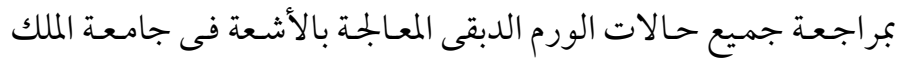

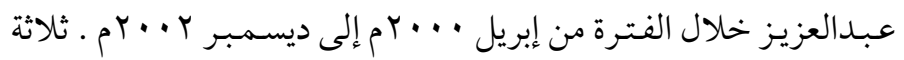

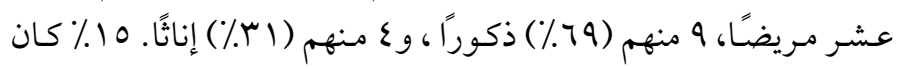

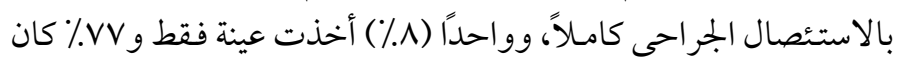

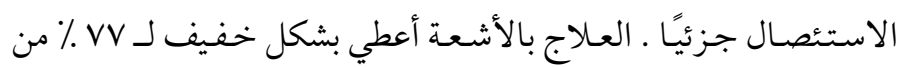

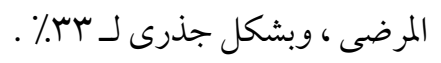

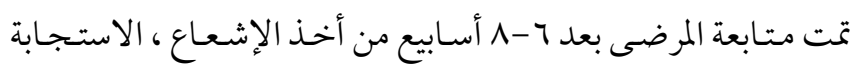

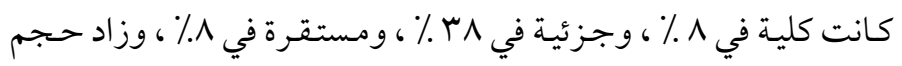

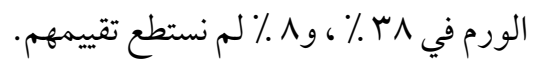

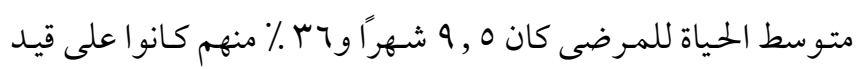
الحياة لمدة سنة . متوسط. 